\title{
Aspectos radiográficos da mandíbula e crista interdentária de bovinos induzidos ao hiperparatiroidismo secundário nutricional
}

\section{Radiographics aspects of mandible and interdentary crest of bovine induced to secondary nutritional hyperparathyroidism}

\author{
Jacinta Eufrásia Brito Leite, ${ }^{*}$ Frederico Celso Lyra Maia, ${ }^{* *}$ Pierre Castro Soares, ${ }^{* *}$ Rosilda Maria Barreto Santos, ${ }^{* *}$ \\ Vera Alvarenga Nunes, ${ }^{* *}$ Lucy Marie Ribeiro Muniz ${ }^{* * *}$
}

\begin{abstract}
Resumo
Quinze novilhos não castrados, mestiços leiteiros, com idades entre oito e 14 meses, aparentemente sadios, foram induzidos ao Hiperparatiroidismo Secundário Nutricional (HSN). Com esta finalidade foram distribuídos cinco animais para cada grupo, constituindo-se três grupos distintos e submetendo-os a três tratamentos. O $\mathrm{T}_{1}$ continha o nível de cálcio (Ca) equivalente a $0,45 \%$ e de fósforo (P) a 0,36\%; o $\mathrm{T}_{2}$ com o nível de Ca igual a 0,45\% e o de $\mathrm{P}$ a $0,72 \%$ e o $\mathrm{T}_{3}$ com o nível de Ca igual a $0,34 \%$ e o de $\mathrm{P}$ a $0,14 \%$. Decorridos 180 dias, os animais foram sacrificados e radiografias da mandíbula foram obtidas. Redução na altura da crista interdentária e da densidade óssea da mandíbula foram os sinais radiográficos mais evidentes, constituindose, desta forma, uma referência radiográfica no diagnóstico de bovinos com HSN.
\end{abstract}

Palavras-chave: hiperparatiroidismo, radiografia, doença metabólica, cálcio, fósforo, bovinos.

\section{Abstract}

Fifteen-crossbreed healthily male herd, aging from 8 to 14 months, were induced to Secondary Nutritional Hiperparathyroidism $(\mathrm{SNH})$. Three groups of five animals were randomly distributed and submitted to three treatments. The control group $\left(\mathrm{T}_{1}\right)$ was treated with $0.45 \%$ of calcium $(\mathrm{Ca})$ and $0.36 \%$ of phosphorus $(\mathrm{P})$; the other group were treated with $0.45 \%$ of $\mathrm{Ca}$ and $0.72 \%$ of $\mathrm{P}\left(\mathrm{T}_{2}\right)$ and with $0.34 \%$ of $\mathrm{Ca}$ and $0.14 \%$ of $\mathrm{P}\left(\mathrm{T}_{3}\right)$. After 180 days from the beginning of the experiment the animals were scarified and radiographs of jaw were performed. The reduction of intermediary crest height and mandible density were the radiographic signs more evident, composing a radiographic reference in bovine with SNH

Keywords: hyperpatathyroidism, X-ray, metabolic diseases, calcium, phosphorus, bovine.

\section{Introdução}

O hiperparatiroidismo secundário nutricional (HSN) é uma patologia que acomete várias espécies domésticas e silvestres. No Brasil, o HSN tem sido relacionado com a doença periodontal (Cara Inchada) dos bovinos (Nunes et al., 1979; Brito, 1983), doença de natureza endêmica em vários estados da federação, notadamente em áreas do cerrado e solos pobres (Souza et al., 1986).

Tanto a deficiência de cálcio $(\mathrm{Ca})$ quanto o excesso de fósforo (P) provocam hipocalcemia, que estimula as glândulas paratiróides. O paratormônio produzido induz a reabsorção óssea, via osteólise, e restabelece a homeostase do $\mathrm{Ca}$ plasmático (Krook, 1988).

Alterações persistentes dos níveis de $\mathrm{Ca}$ e $\mathrm{P}$ plasmáticos induzem HSN e sua conseqüência é a osteopenia generalizada, embora os ossos não sejam uniformemente afetados.
Em relação à hierarquia da diminuição da densidade óssea, em ordem decrescente, ocorre em maior intensidade nos ossos maxilares, notadamente o processo alveolar, seguindo-se outros ossos do crânio, costelas, vértebras e ossos longos (Krook, 1976).

Clinicamente, o HSN se manifesta nos estágios mais avançados por abaulamento dos ossos faciais, amolecimento e perda de dentes, dificuldade de mastigação, emagrecimento progressivo e, em alguns casos, morte. O diagnóstico clínico precoce desta doença é muito difícil, pois quando as lesões tornam-se evidentes, o processo já está avançado, dificultando ou mesmo inviabilizando o tratamento; além de que análises bioquímicas do sangue freqüentemente apresentam níveis de $\mathrm{Ca}$ e $\mathrm{P}$ normais, devido à ação do paratormônio. Importante considerar a busca por possibilidades de melhor diagnosticar uma determinada enfermidade e, neste caso, a avaliação radiográfica dos ossos gnáticos pode ser um valioso recurso no diagnóstico do HSN.

\footnotetext{
* Professora MSc do Departamento de Medicina Veterinária da Universidade Federal Rural de Pernambuco (UFRPE). Rua Dom Manuel de Medeiros, s/n, Dois Irmãos, Recife, PE. CEP 52171-900

** Professor Dr. do Departamento de Medicina Veterinária da UFRPE

***Professora Dra. da Escola de Veterinária da Universidade Federal de Minas Gerais

****Professora Dra. da Faculdade de Medicina Veterinária e Zootecnia - Unesp - Botucatu
} 
Este trabalho tem por objetivo descrever os achados radiográficos da mandíbula e crista interdentária em bovinos jovens induzidos ao HSN, sendo estes alimentados com diferentes teores de cálcio e fósforo dietéticos.

\section{Material e métodos}

Foram utilizados 15 novilhos não castrados, mestiços leiteiros, com idades entre oito e 14 meses, aparentemente sadios. Os animais foram mantidos em instalações apropriadas, em sistema de confinamento, alojados individualmente em baias dotadas de cocho e bebedouro, pelo período de 180 dias. Os mesmos foram tratados, mensalmente, com complexo ADE, recebendo $1 \mathrm{ml} / 50 \mathrm{~kg}$ do peso vivo (Laboratório HERTAPE), durante a experimentação.

Os animais foram distribuídos aleatoriamente em três lotes de cinco, recebendo dieta isoprotéica, isocalórica e com a mesma composição mineral, segundo o National Research Council NRC (1996), exceto quanto ao Ca e ao P, que sofreram alterações quantitativas para constituírem os seguintes tratamentos:

Tratamento $1\left(\mathrm{~T}_{1}\right)$ - Níveis quantitativos de $\mathrm{Ca}$ e $\mathrm{P}$ recomendados pelo NRC;

Tratamento $2\left(\mathrm{~T}_{2}\right)$ - Níveis quantitativos de $\mathrm{P}$ superior e o de $\mathrm{Ca}$ igual ao recomendado pelo NRC;

Tratamento $3\left(\mathrm{~T}_{3}\right)$ - Níveis de Ca e $\mathrm{P}$ abaixo do recomendado pelo NRC.

A dieta dos animais constituiu-se de uma ração composta de feno de Brachiaria ruziziensis, triturado e passado em peneira de $1 \mathrm{~cm}$ de diâmetro, complementada por outros ingredientes (Quadro 1). Os níveis de Proteína Bruta, Energia metabolizável, Ca e $\mathrm{P}$ na Matéria Seca dos trata-mentos também estão relacionados na Tabela 1.

A dieta experimental foi fracionada, sendo fornecida duas vezes ao dia (manhã e tarde). As sobras foram pesadas, de modo a se obter o consumo diário real. A quantidade diária total da ração foi calculada com base no consumo médio diário da semana anterior, acrescido de $15 \%$ da mesma. A água foi consumida ad libitum.

Todos os animais receberam, durante os dez primeiros dias antecedentes ao experimento, a ração do Tratamento 1 e foram submetidos a exame clínico e medidas sanitárias de rotina.

Decorridos 180 dias do experimento, os animais foram sacrificados por eletrocussão. As mandíbulas foram dissecadas e preparadas para radiografias, sendo estas realizadas na projeção látero-lateral, totalizando 30 radiografias. A exposição utilizada foi de 70 quilivolts (KV) e 80 miliamperes/segundo (mAs). O tempo de exposição foi de 0,06 segundos e a distância foco-filme foi de $1,10 \mathrm{~m}$. Após revelação pelo método de rotina (revelador, enxágüe, fixador e lavagem), as radiografias permaneceram na secadora por cinco minutos.

As radiografias foram interpretadas e descritas em protocolos individuais. Para a variável densidade óssea mandibular e visualização da lâmina cortical das cristas interdentárias, os achados radiográficos foram registrados conforme os respectivos escores:

0 - Sem alteração;

1 - Discreta diminuição de densidade óssea e estreitamento da cortical, além de pequenos pontos de rarefação óssea;
2 - Moderada diminuição de densidade e estreitamento da cortical, além de moderadas áreas de rarefação óssea;

3 - Severa diminuição de densidade óssea e estreitamento da cortical, além de grandes áreas de rarefação óssea.

1 - Total visualização da lâmina cortical das cristas interdentárias;

2 - Parcial visualização da lâmina cortical das cristas interdentárias.

Quadro 1: Composição das rações oferecidas aos animais em seus respectivos tratamentos

\begin{tabular}{|c|c|c|c|}
\hline COMPOSIÇÃO & $\mathrm{T} 1$ & T 2 & T 3 \\
\hline Brachiaria ruziziensis (feno) (\%) & 79,33 & 77,79 & 80,42 \\
\hline Farelo de soja (\%) & 16,83 & 16,83 & 16,83 \\
\hline Óleo de soja (\%) & 2,65 & 2,65 & 2,65 \\
\hline Carbonato de cálcio (\%) & 0,33 & 0,33 & - \\
\hline Fosfato monobásico de sódio (\%) & 0,76 & 2,30 & - \\
\hline Sal traço - mineralizado* $(\%)$ & 0,10 & 0,10 & 0,10 \\
\hline Proteína bruta (\%) & 12,60 & 12,50 & 12,67 \\
\hline Energia Metabolizável $(\mathrm{Mkal} / \mathrm{kg})^{1}$ & 2,51 & 2,47 & 2,53 \\
\hline Cálcio (\%) & 0,45 & 0,45 & 0,34 \\
\hline Fósforo (\%) & 0,36 & 0,72 & 0,18 \\
\hline
\end{tabular}

Para a análise estatística, utilizou-se o teste de Kruskal-Wallis, para avaliar diferenças entre os tratamentos, e o teste $t$ de Student para comparação dos postos no teste de KruskalWallis, em relação às variáveis: densidade óssea mandibular e visualização da lâmina cortical das cristas interdentárias.

Para observar a existência de associação entre a visualização total e parcial da lâmina cortical das cristas interdentárias (entre os dentes PM3-M1, M1-M2 e M2-M3), substituição dos pré-molares e perda dos ápices da lâmina cortical com os diferentes níveis de cálcio e fósforo nas dietas, foi aplicado o teste exato de Fisher. Já em relação às freqüências da visualização total e parcial da lâmina cortical das cristas interdentárias, dentro de cada tratamento, realizou-se teste de diferença entre proporções. Correlações entre as diferentes variáveis estudadas foram estimadas pelo cálculo do coeficiente de Spearman $\left(r_{s}\right)$, segundo Sampaio (1998).

\section{Resultados}

Não se observou diferenciação da soma dos postos na análise de densidade óssea mandibular, porém uma menor capacidade de visualização da lâmina cortical das cristas interdentárias nos animais do T2 foi significativa em relação ao T1 e T3 (Tabela 1; Figuras 1 e 2).

Tabela 1: Distribuição dos escores e soma dos postos, por tratamento, da análise radiográfica da mandíbula e crista interdentária dos bovinos induzidos ao HSN

\begin{tabular}{ccc}
\hline Tratamentos & \multicolumn{2}{c}{ Soma dos Postos } \\
\cline { 2 - 3 } & DOM & $\mathrm{LCCl}^{2^{* *}}$ \\
\hline T1 & $5,7^{\mathrm{a}}$ & $10,5^{\mathrm{a}}$ \\
T2 & $8,7^{\mathrm{a}}$ & $4,5^{\mathrm{b}}$ \\
T3 & $9,6^{\mathrm{a}}$ & $9,0^{\mathrm{a}}$ \\
\hline
\end{tabular}

$\mathrm{X}_{2 \mathrm{gl}}=5,99 ;{ }^{1} \mathrm{~T}=2,2893 ;{ }^{2} \mathrm{~T}=7,2834$

"Densidade Óssea mandíbulas; "* Visualização da lâmina cortical das cristas interdentárias. 


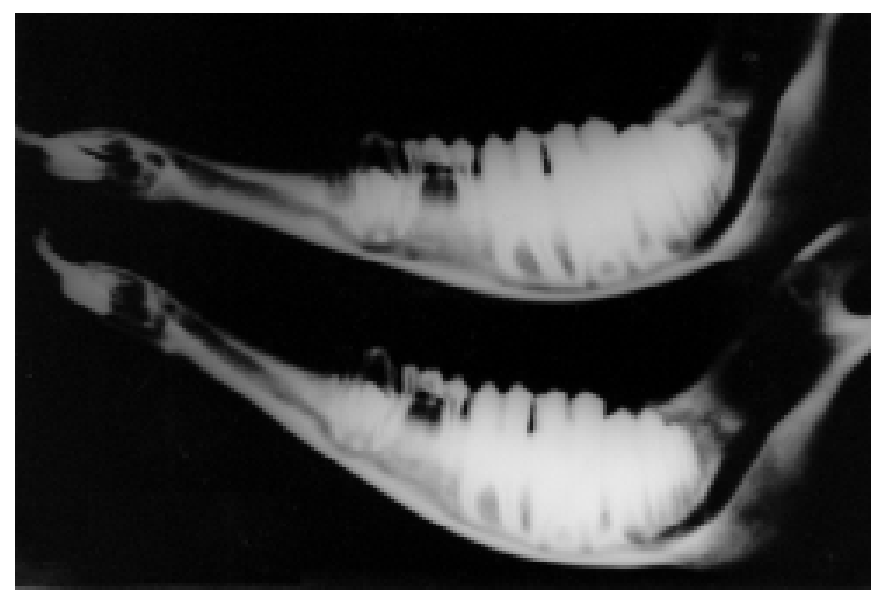

Figura 1: Radiografia em incidência lateral da mandíbula de bovino pertencente ao T1, evidenciando moderada diminuição da densidade óssea, além das cristas interdentárias (setas).

$\mathrm{Na}$ Tabela 2, não foi possível identificar diferenças dos contrastes das cristas (PM3-M1, M1-M2 e M2-M3) entre os tratamentos, em relação à capacidade de visualização total ou parcial da lâmina cortical das cristas interdentárias. No entanto, verifica-se que maior variação da capacidade de visualização total para parcial foi observada nos animais do T2.

Tabela 2: Distribuição das freqüências entre pares de dentes, por tratamento, pela análise radiográfica para visualização total e parcial da lâmina cortical das cristas interdentárias dos bovinos induzidos ao HSN

\begin{tabular}{ccccccc}
\hline \multirow{2}{*}{$\begin{array}{c}\text { Tratamentos/ } \\
\text { Interações }\end{array}$} & \multicolumn{2}{c}{$\mathrm{PM}-\mathrm{M} 1$} & \multicolumn{2}{c}{$\mathrm{M} 1-\mathrm{M} 2$} & \multicolumn{2}{c}{$\mathrm{M} 2-\mathrm{M} 3$} \\
\cline { 2 - 7 } & Total & Parcial & Total & Parcial & Total & Parcial \\
\hline T1 & 5 & 0 & 5 & 0 & 5 & 0 \\
T2 & 2 & 3 & 3 & 2 & 4 & 1 \\
T3 & 5 & 0 & 3 & 2 & 5 & 0 \\
\hline T1 $x$ T2 & $\mathrm{p}=0,0833$ & $\mathrm{p}=0,2222$ & $\mathrm{p}=0,5000$ \\
T1 × T3 & $\mathrm{p}=1,0000$ & $\mathrm{p}=0,2222$ & $\mathrm{p}=1,0000$ \\
T2 x T3 & $\mathrm{p}=0,0833$ & $\mathrm{p}=0,7384$ & $\mathrm{p}=0,5000$ \\
\hline
\end{tabular}

Já na Tabela 3, considerando a soma dos postos da Tabela 2, o efeito tratamento foi observado na contingência T1 $\times$ T2, com variação significativa $(p=0,0084)$. Com relação à análise de comparação entre pares de proporção, dentro de cada tratamento, variação significativa foi identificada no $\mathrm{T} 1\left(\mathrm{X}^{2}\right.$ calc $=15,03)$ e no T3 $\left(X^{2}=8,06\right)$. Verifica-se, também, que no $\mathrm{T} 2$, com maior teor de $\mathrm{P}$, maior é o número de animais com menor capacidade de visualização da LCCI.

Avaliando-se a associação entre tratamentos e variáveis radiográficas dos dentes dos bovinos (Tabela 4), a variação foi significativa da substituição dos pré-molares, na qual se verifica maior freqüência no T2 $(p=0,0041)$ e T3 $(p=0,0238)$. Tal observação é confirmada com a análise dos contrastes entre pares de tratamentos. Em relação à perda dos ápices das lâminas corticais, verifica-se uma significância entre $T 1 \mathrm{x}$ T2 $(p=0,0238)$.

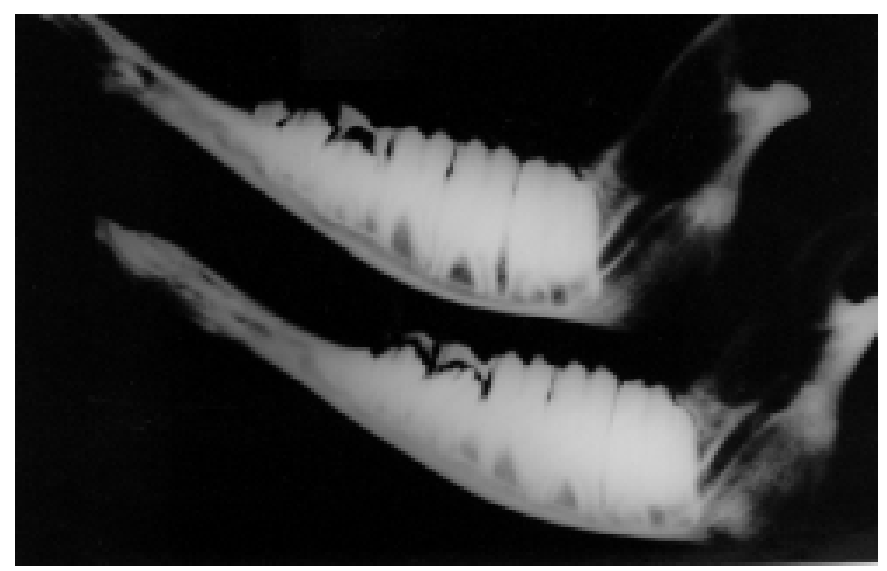

Figura 2: Radiografia em incidência lateral da mandíbula de bovino pertencente ao T2, evidenciando severa diminuição da densidade óssea e altura das cristas interdentárias (setas).

Tabela 3: Freqüências das somas dos postos entre dentes, por tratamento, da análise radiográfica da visualização da lâmina cortical das cristas interdentárias de bovinos induzidos ao HSN

\begin{tabular}{|c|c|c|c|}
\hline \multirow[b]{2}{*}{ Tratamentos } & \multicolumn{2}{|c|}{$\mathrm{LCCl}$} & \multirow{2}{*}{$\begin{array}{c}\text { Contraste entre pares } \\
\text { de tratamento }\end{array}$} \\
\hline & Total & Parcial & \\
\hline T1 & $15(100 \%)^{a}$ & $0(0 \%)^{b}$ & $\begin{array}{c}\text { T1 } x \text { T2 } p=0,0084^{* *} \\
X^{2}{ }_{\text {calc. }}>X^{2}{ }_{\text {Tab. }}^{* *}\end{array}$ \\
\hline T2 & $9(60 \%)^{a}$ & $6(30 \%)^{a}$ & $\begin{array}{c}\text { T1 } \mathrm{xT3} p=0,2414 \\
\mathrm{X}^{2}{ }_{\text {calc. }}<\mathrm{X}^{2}{ }_{\text {Tab. }}\end{array}$ \\
\hline T3 & $13(86,67 \%)^{a}$ & $2(13,33 \%)^{b}$ & $\begin{array}{c}\text { T2xT3 } p=0,1074 \\
X^{2}{ }_{\text {calc. }}>\mathrm{X}^{2}{ }_{\text {Tab. }}\end{array}$ \\
\hline
\end{tabular}

- Visualização da lâmina cortical das cristas interdentárias

" Teste Exato de Fisher para pares de tratamentos

** Teste de diferença entre proporções para o fator LCCI total e parcial. Letras minúsculas não-coincidentes na mesma linha diferem entre si ao nível de $5 \%$ de probabilidade

Tabela 4: Dispersão de freqüências das variáveis com respostas dicotômicas nas análises radiográficas dos dentes dos bovinos induzidos ao HSN

\begin{tabular}{crrrr}
\hline \multirow{2}{*}{$\begin{array}{c}\text { Tratamentos/ } \\
\text { Interações }\end{array}$} & \multicolumn{2}{c}{$\begin{array}{c}\text { Substituição dos Pré- } \\
\text { molares }\end{array}$} & \multicolumn{2}{c}{$\begin{array}{c}\text { Perda dos ápices da } \\
\text { lâmina cortical }\end{array}$} \\
\cline { 2 - 5 } & Não & Sim & Sem & Com \\
\hline T1 & 5 & 0 & 5 & 0 \\
T2 & 0 & 5 & 1 & 4 \\
T3 & 1 & 4 & 3 & 2 \\
\hline T1 x T2 & $\mathrm{p}=0,0041^{*}$ & $\mathrm{p}=0,5000$ \\
T1 x T3 & $\mathrm{p}=0,0238^{*}$ & \multicolumn{2}{c}{$\mathrm{p}=0,0238^{\star}$} \\
T2 x T3 & $\mathrm{p}=0,5000$ & \multicolumn{2}{c}{$\mathrm{p}=0,2620$} \\
\hline
\end{tabular}

* Significativa ao nível de $p<0,05$ 
Correlações positivas e significativas entre os pares de variáveis foram observadas (Tabela 5).

Tabela 5: Coeficiente de correlação de postos de Spearman $\left(r_{s}\right)$ de variáveis oriundas da análise radiográfica da mandíbula e dentes de bovinos induzidos ao HSN

\begin{tabular}{ccccc}
\hline Variáveis & $\mathrm{DOM}^{1}$ & $\mathrm{LCCl}^{2}$ & $\mathrm{SPM}^{3}$ & $\mathrm{PALC}^{4}$ \\
\hline DOM & 1 & $0,53^{*}$ & $0,56^{*}$ & $0,58^{*}$ \\
LCCl & & 1 & $0,81^{\star *}$ & $0,98^{\star *}$ \\
SPM & & & 1 & $0,88^{\star *}$ \\
PALC & & & & 1
\end{tabular}

Densidade óssea mandibular; ${ }^{2}$ lâmina cortical das cristas interdentárias; ${ }^{3}$ Substituição dos pré-molares; ${ }^{4}$ Perda dos ápices da lâmina cortical

* $p<0,05 ; "$; $<0,0001$

\section{Discussão}

Neste estudo verificou-se que as lesões indicavam possuir caráter sistêmico, o que corrobora as afirmativas de Nunes e Chiquiloff (1986), Nunes et al. (1995) e Maia (1995). Observou-se, ainda, que estas lesões eram mais evidentes e de maior intensidade nos animais pertencentes ao $T_{2}$ e $T_{3}$, devido aos diferentes teores de $\mathrm{Ca}$ e $\mathrm{P}$ nas dietas.

Para ruminantes, consideram-se satisfatórios os níveis de Ca e P de 2:1 (Andriguetto et al., 1985; NRC, 1996). Variações de elementos minerais, conforme visto no T2 e T3 induzem a ação do hormônio da paratiróide (PTH), estimulando a osteólise osteocítica e osteoclástica, aumentando a quantidade de osteoclastos nas superfícies ósseas (Santos, 1992).

As observações radiográficas encontradas neste estudo são compatíveis com os achados de Maia (1990), o qual analisou histopatologicamente as paratiróides e os ossos alveolares destes animais, confirmando que diferenças de níveis Ca e P oferecidos nas dietas são responsáveis pelo desenvolvimento do HSN, onde a osteólise osteocítica provocou reabsorção do osso alveolar, expressa radiograficamente pela presença de diminuição de densidade óssea nas cristas interdentárias, promovendo uma diminuição da visualização das lâminas corticais destas cristas (Tabelas 1, 2 e 3) e perda dos ápices da lâmina cortical (Tabela 4), como confirmado pela maior soma dos postos para LCCI, bem como pelas maiores freqüências da capacidade de visualização parcial das lâminas corticais dos animais do T2.

O fato das alterações terem sido mais evidentes no T2, Krook (1988) e Palmer (1993), confirma que o excesso de P na dieta é mais eficiente em induzir a hiperfunção paratiróide do que a deficiência de Ca.

$\mathrm{Na}$ soma dos postos da variável densidade óssea mandibular, não se verificou variação significativa entre tratamentos, embora se perceba que as maiores somas dos postos foram para o T2 e T3.

Divergindo dos achados radiográficos de Brito (1983), neste estudo não foi observado, aos 180 dias do experimento, ne- nhum bovino com perda de dentes, observando-se a substituição dos mesmos por se tratar de animais jovens. Possivelmente com um tempo mais longo de experimentação, aquele achado pudesse ser confirmado, em conseqüência da constante desmineralização óssea, principalmente nos casos de animais submetidos a um desequilíbrio mineral mais acentuado, como no caso do T2 e T3. Henrikson (1968) submeteu cães sadios ao HSN e observou que as lesões progrediam significativamente com o tempo.

Como visto na tabela 4 , as maiores freqüências para substituição dos pré-molares foram para o T2 e T3. Isto pode ser justificado pelo fato de que manifestações de osteodistrofia fibrosa ocorrem em bovinos com HSN, cujo quadro osteopênico, secundário à hiperfunção paratiróidea, propiciar uma precoce substituição dos pré-molares, conseqüência do comprometimento dos tecidos de sustentação dos dentes (Nunes et al., 1979; Brito, 1983; Soni, 1984).

Decorridos os 180 dias da indução, Maia et al. (1995) observaram ao nível do periodonto, que a perda do osso alveolar encontrava-se bem evidenciada, com fragmentação e acinturamento das trabéculas, com formação de grandes cavidades de reabsorção e intensa osteoclasia, sendo, estes animais, pertencentes ao T2.

Soni (1984) observou regressão dos sinais de osteopenia e rarefação nas cristas interdentárias com a oferta de rações com níveis de $\mathrm{Ca}$ e $\mathrm{P}$ equilibrados aos animais portadores de HSN. No presente experimento a osteopenia foi provocada, demonstrando diminuição de densidade óssea mandibular, com menores escores para o T2 e T3 e crista interdentária, também relacionados ao desequilíbrio e carência dos mesmos elementos minerais na ração diária. Observou-se que no tocante ao osso inicialmente afetado pela diminuição de densidade, pôde-se corroborar a afirmativa de Krook (1976), quando relatou que a osteopenia tem início nos ossos maxilares e que o processo alveolar é o mais precocemente afetado. Coincide, também, com os achados de Saville et al. (1969), que descreveram menor capacidade de visualização da lâmina cortical dos dentes como achado radiográfico do HSN.

Estas observações são consideradas importantes, uma vez que a análise radiográfica, tanto da densidade óssea mandibular quanto dos dentes de bovinos com HSN, servem de subsídios relevantes para avaliação clínica de animais que desenvolvem este tipo de problema, em conseqüência dos desequilíbrios dos elementos minerais constituintes de dietas para animais de interesse pecuário, além de servirem como indicadores clínicos importantes para o diagnóstico desta enfermidade.

Os resultados permitem concluir que dietas com excesso de fósforo promovem maior grau de diminuição da densidade óssea mandibular, com estreitamento de cortical e áreas de rarefação óssea, além de que maior é a substituição dos prémolares, perda da capacidade de visualização da lâmina cortical das cristas interdentárias e perda do seu ápice. A análise radiográfica de ossos mandibulares e dentes pode ser utilizada como elemento diagnóstico do HSN em bovinos. 


\section{Referências}

ANDRIGUETTO, J. M.; PERLY, L.; MINARDI, J. et al. Nutrição animal. As bases e os fundamentos da nutrição animal. 4. ed. São Paulo: Nobel, 1985. $395 \mathrm{p}$.

BRAZ, M. B. Semiologia médica animal. Lisboa: Fundação Calouste Gulbenkian, 1972, v. 2, p. 317-318.

BRITO, B. A L. Aspectos morfológicos da doença periodontal em bovinos. 1983. 51 f. Tese (Mestrado)-Escola de Medicina Veterinária - Universidade Federal de Minas Gerais, 1983.

HENRIKSON, P. Periodontal disease and calcium deficiency: an experimental study in the dog. Acta Odontol. Sccand., v. 26, p. 1-132, 1968.

KEALY, R. D. Calcium metabolism. Canine pratice, v. 4, n. 1, p. 1-3, 1977.

KROOK, L. Periodontal disease in dog and man. Adv. Vet. Sci. Comp. Med., v. 20, p. 171-190, 1976.

KROOK, L. Doenças metabólicas do osso. Cad. Téc. Vet. UFMG, Belo Horizonte, v. 3, p. 1-66, 1988.

MAIA, F. C. L.; NOGUEIRA, R. H. G.; NUNES, V. A.; NUNES, I. J. Hiperparatireoidismo secundário nutricional em bovinos: 1. Morfologia e morfometria das paratireóides. Arq. Bras. Med. Vet. Zootec., v. 47, n. 3, p. 329-341, 1995.

NATIONAL RESEARCH COUNCIL-NRC. Nutrient requeriment of beef cattle. 7. ed. Washington D. C., 1996, 242 p.
NUNES, V. A; CHIQUILOFF, M. A. G. Doença periodontal ou "Cara Inchada" do bovino. Cad. Tec. Esc. Vet. UGMG, Belo Horizonte, v. 1, p.3-8, 1986.

NUNES, V. A.; MAIA, F. C. L.; NOGUEIRA, R. H. G.; NUNES, I. J.; CARDOSO, E. C. Hiperparatireoidismo secundário nutricional em bovinos: 2. Morfologia do periodonto. Arq. Bras. Med. Vet. Zootec., v. 47, n. 3, p. 343-360, 1995.

PALMER, N. Bones and Joints. In: JUBB, K. V. F.; KENNEDY, P. C.; PALMER, N. Pathology of domestic animals. 4. ed., San Diego: Academic Press, 1993. v. 1, p. 1-181.

SAMPAIO, I.B.M. Estatística aplicada à experimentação animal. Belo Horizonte: Fundação de Amparo à Pesquisa em Medicina Veterinária e Zootecnia, 1998.

SANTOS, R.S.V. Nutrição e principais enfermidades ósseas em cães jovens. Cães e Gatos, n. 42, p. 9-18, 1992.

SAVILLE, P.D.; KROOK, L.; GUSTAFSSON, P. O et al. Nutritional secundary hiperparathyroidism in a dog morfologic and radioisotope studies with treatment. Cornell Veterinarian, v. 59, n. 1, p. 155-167, 1969.

SONI, C. A. Influência do cálcio, do fósforo e do cobre na "doença periodontal'. 1984. 46 f. Tese (Mestrado)-Escola de Medicina Veterinária-Universidade Federal de Minas Gerais, 1984.

YATES, D.F.; HUNT, E. Distúrbio do metabolismo do cálcio. In: SMITH, B. P. Tratado de Medicina Interna de Grandes Animais: moléstias de eqüinos, bovinos, ovinos e caprinos. Rio de Janeiro: Guanabara Koogan, 1994. v. 2, p. 1306-1312. 